\title{
Justification of the Shape of a Non-Circular Cross-Section for Drilling With a Roller Cutter
}

\author{
Gennady Buyalich ${ }^{1 *}$, and Mikhail Husnutdinov ${ }^{1}$ \\ ${ }^{1}$ T.F. Gorbachev Kuzbass State Technical University, 650000, 28 Vesennyaya St., Kemerovo, Russia
}

\begin{abstract}
The parameters of the shape of non-circular cross-section affect not only the process of blasting, but also the design of the tool and the process of drilling as well. In the conditions of open-pit mining, it is reasonable to use a roller cutter to produce a non-circular cross-section of blasting holes. With regard to the roller cutter, the impact of the crosssection shape on the oscillations of the axial force arising upon its rotation is determined. It is determined that a polygonal shape with rounded corners of the borehole walls connections and their convex shape, which ensures a smaller range of the total axial force and the torque deflecting the bit from the axis of its rotation is the rational form of the non-circular cross-section of the borehole in terms of bit design. It has been shown that the ratio of the number of cutters to the number of borehole corners must be taken into account when justifying the shape of the cross-section, both from the point of view of the effectiveness of the explosion action and from the point of view of the rational design of the bit..
\end{abstract}

\section{Introduction}

The non-circular shape of the cross-section, as shown by a number of studies [1-11], affects the process of explosive crushing of rocks. To create an elongated cavity in a rock with a non-circular cross-section, appropriate drilling tools are created [12]. For drilling boreholes in open-pit mining operations, it is suggested to use roller cutters in order to form a non-circular cross-section, and the effect of the cross-sectional shape on both the effectiveness of the explosion action and on the design of the roller cutter and the drilling process must be considered.

\section{Materials and methods}

The non-circular shape of the cross-section with stress raisers is presented in variants with notches on the walls of the bore-hole, or as a polygonal cross-section whose angles are also stress raisers. In the latter case, the area of the lateral surface, which is related to the crosssectional area, changes, which causes an additional effect. Proceeding from this, the criteria for the cross-sectional shape are formulated from the point of view of the effectiveness of the explosion: the relative range of the radii of the cross-section, the connecting angle of the

${ }^{*}$ Corresponding author: gdb@kuzstu.ru 
walls of the cavity, the increase in the area of the lateral surface of the well with a noncircular cross-section.

The relative range of the radii tends to the maximum with increasing concavity of the walls of the well, while the connecting angle of the walls of the borehole decreases and the area of their lateral surface increases. According to these criteria, the best, in terms of the effectiveness of the explosion, shapes of the cross-section are those with a smaller number of angles and a concave wall shape.

Therefore, biangular, triangular and quadrangular shapes of cross-section with rectilinear and curvilinear shape of the walls of the borehole are considered below (Fig. 1).

The possibility of forming a cross-section in the form of a regular figure by single-cone roller cutters is proved. The number of angles is determined by the gear ratio of the roller bit $i$, and by the shape of the roller cutter (Fig. 2). It is determined that with the help of bits having one, two, three cutters, it is possible to produce blast holes with a concave shape of their face and a regular shape of the cross-section having rectilinear, convex and concave sections of the sides, except for the shape in the form of a regular triangle that can only be obtained by one roller cutter. For rectilinear sides of a polygonal cross-section or with the same curvature, the rational shape of the cross-section from the point of view of the constructive perfection of the tool is the shape of the cross-section with rounded angles, and a larger curvature at which the maximal permissible cone angle of the cutter increases by the condition of its free rotation inside the well.

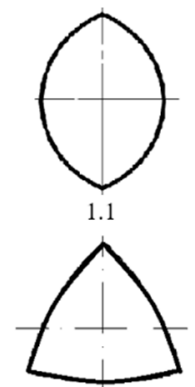

2.1

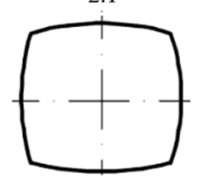

3.1

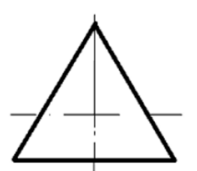

2.2

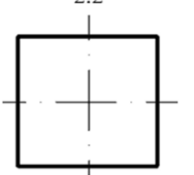

3.2

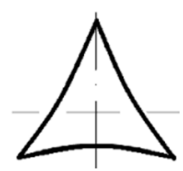

2.3

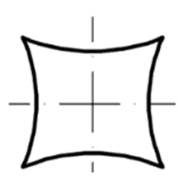

3.3

Fig. 1. Variants of shapes of the bore-hole cross-section: 1.1 - biangular; 2.1 - triangular with convex sides; 2.2 - triangular with rectilinear sides; 2.3 - triangular with concave sides; 3.1 - quadrangular with convex sides; 3.2 - quadrangular with rectilinear sides; 3.3 - quadrangular with concave sides.

To obtain a non-circular cross-section, it is proposed to form the base of the circular cone of cutters by planes intersecting along the line of its rotation diameter and passing at an acute angle to its axis (Fig. 3). In this case, with the concave shape of the bottom, the cone angle of the cutter is not limited by the condition of its free rotation inside the well and at an angle greater than or equal to 60 degrees, rolling the cutter along the bottom reproduces the convex sides of the walls of the well of different curvature, and if the angle is less than 60 degrees, the concave elements of the walls of the well of different curvature (Fig. 4). 


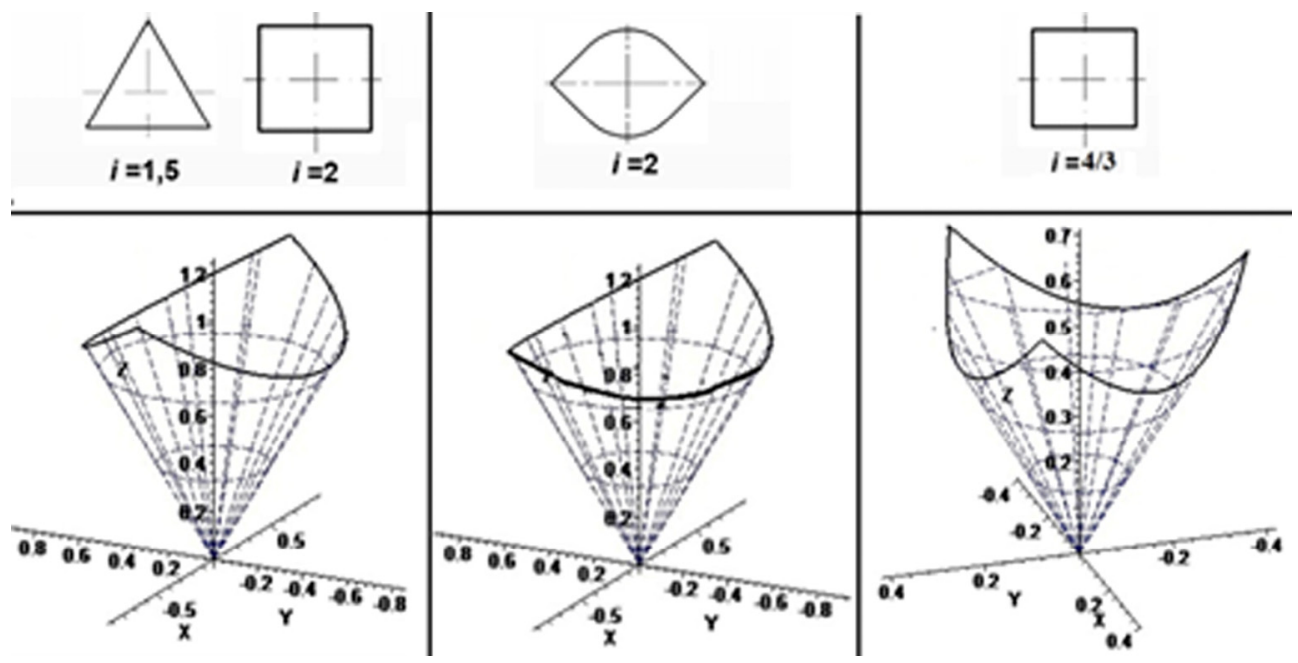

Fig. 2. Concordance of cross-section shapes, gear ratio of the bit $i$ and the roller cutter shape
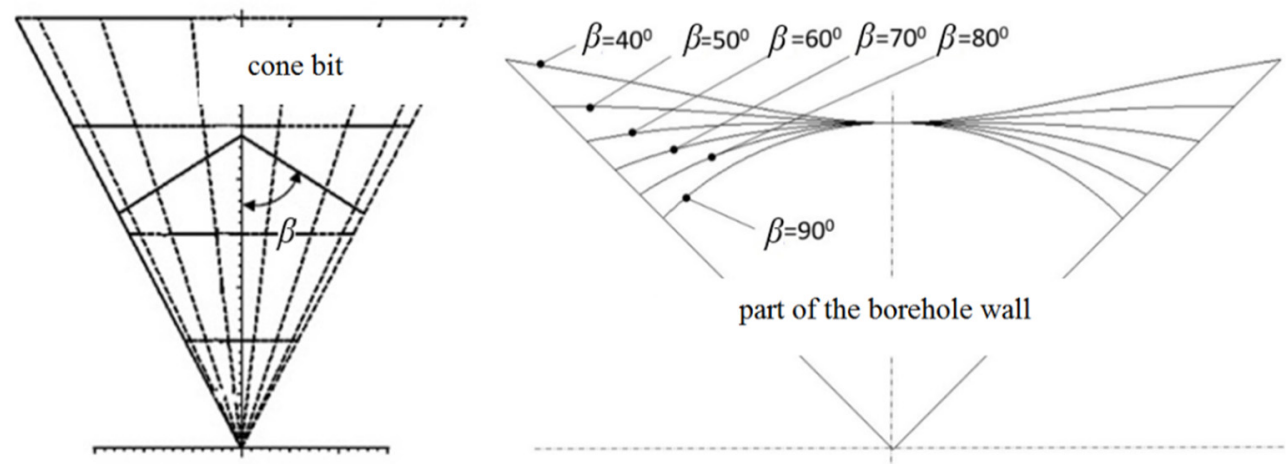

Fig. 3. Forming convex and concave sections of the bore hole wall depending on angle $\beta$ between the axle of the cutter cone and the planes of its shear.
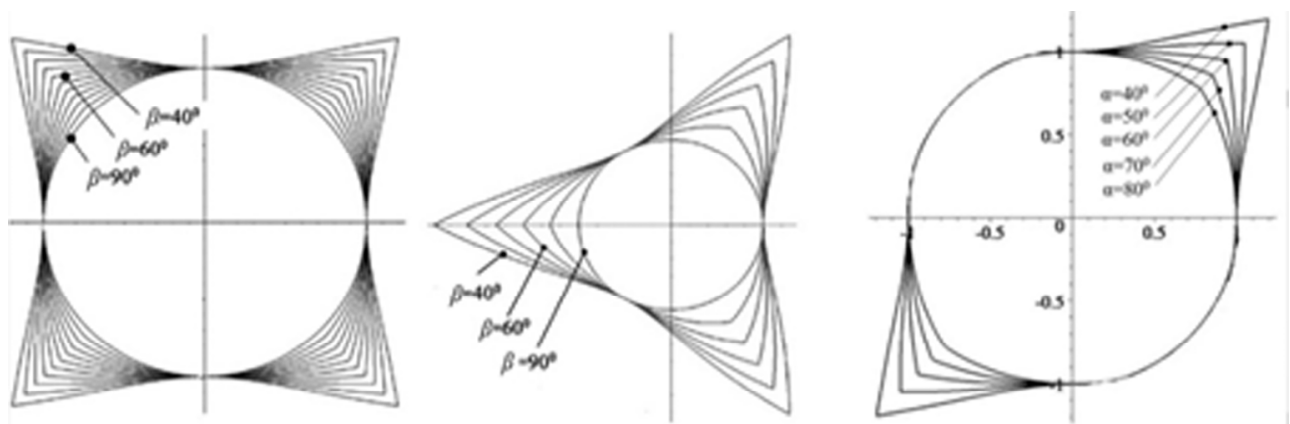

Fig. 4. Impact of angle $\beta$ on shaping various cross-sections.

To assess the impact of the cross-section shape on the force parameters of the roller cutter drilling process, it is assumed that the cutters present a smooth cone that rolls along a smooth, non-deformable bottom of the hole. Such an assumption helps to assess the change in axial forces during tool rotation caused by a non-circular cross-sectional shape without determining the quantitative parameters of the rock reaction. In this case, the reaction forces of the rock are proportional to the contact length of the cutters (Fig. 5). 
The relative range of the total length of the cutter contact with the bore-hole bottom, due to the non-circular cross-sectional shape, decreases with the convex shape of the bore-hole and a greater rounding of the angles of their connection, the fractional ratio of the number of cutters (more than one) to the number of bore-hole corners and the number of cutters greater than the number of bore-hole corners (Fig. 6).

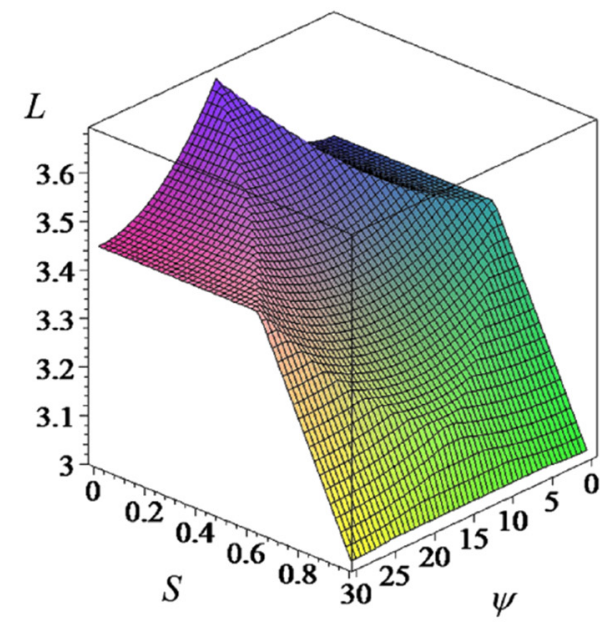

$\mathrm{a}$

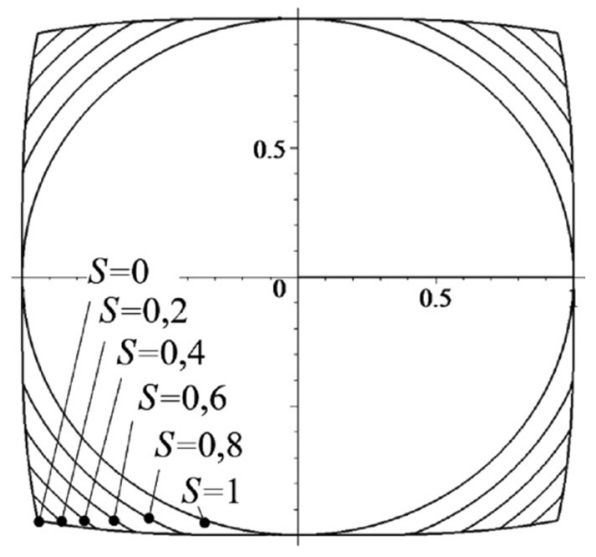

$\mathrm{b}$

Fig. 5. The impact of the degree of rounding the angles $(S)$ of the cross-section of a quadrangular bore-hole at a shear angle $\alpha=60^{\circ}$ and a unit radius of inscribed circumferential $r=1$ on: a - the total length of the contact line $(L)$ of three cutters with the bore-hole bottom depending on the angle of the tool rotation $(\psi) ; \mathrm{b}$ - the shape of the bore-hole cross-section.

It is determined that in the case of fractional multiplicity of the number of several cutters and the angles of the bore-hole cross-section, the non-circular shape of the crosssection stipulates the appearance of the torque deflecting from the axis of cutter rotation (Figure 7). The magnitude and frequency of the change in this deflecting torque correlates with the magnitude and frequency of the change in the range of the axial force.

\section{Results and discussion}

Thus, the parameters of the bore-hole with the non-circular cross-section formed by cutters, including the range of the radii, the angle of the walls connection, and the increase in the area of the lateral surface, are determined by the design parameters of the tool - the gear ratio, the number of cutters, their cone angle and the shape of the gage edge. The total axial force from the bore-hole bottom reactions proportional to the length of the contact line between the cutters and the bottom, and the modulus of the main torque arising from their action that deflects the bit from the axis of its rotation are determined by the parameters of the shape of the non-circular cross-section of the bore-hole and the design parameters of the tool; having the convex form of the bore-hole and a greater rounding of the angles of their connection, a fractional ratio of the number of cutters (more than one) to the number of corners of the well and with the number of cutters greater than the number of corners of the bore-hole, their range stipulated by the non-circular shape of the cross-section decreases. 


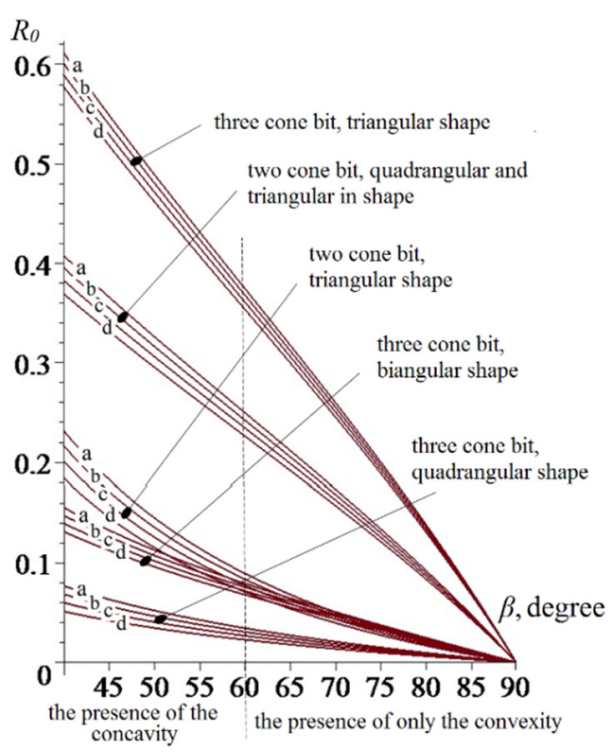

Fig. 6. Relative range $R_{0}$ of the total length of the cutters contact with the bottom depending on the shape of the bore-hole, angle $\beta$ at $S:$ a) 0 ; b) 0.05 ; c) 0.1 ; d) 0.15 .
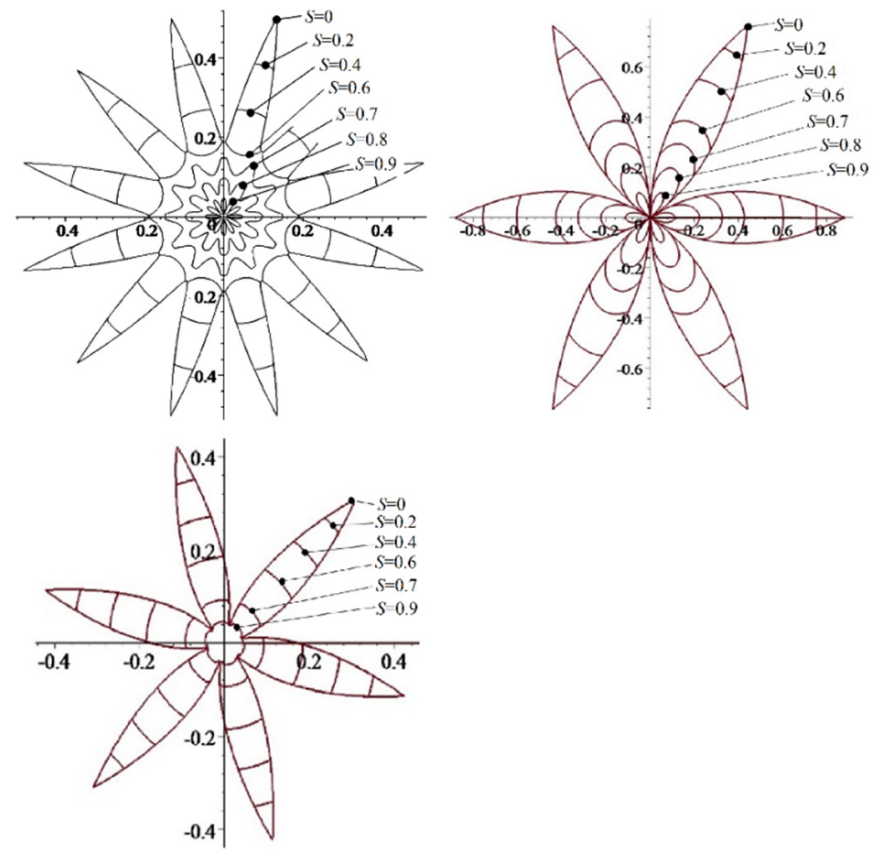

Fig. 7. Change of the modulus of the main torque $(H \cdot \mathrm{m})$ deflecting the bit from its rotation axis due to the degree of rounding of $S$ at $\beta=60^{\circ}, r=1$, angle of inclination of the concave bottom 8 degrees for: a) a three-cone drill bit and quadrangular cross-section; б) a two-cone drill bit and triangular crosssection; в) a three-cone drill bit and biangular cross-section.

\section{Conclusions}


Considering that the range of the axial force and the torque deflecting the bit from its rotation axis are the source of the oscillation processes in the "drill bit - drill pipes drilling rig" system, the preferred shape of the non-circular cross-section of the borehole from the point of view of the rational design of the bit is the polygonal shape with rounded corners of the borehole wall connection and their convex shape, this ensures a smaller range of the total axial force and the torque deflecting the bit from the axis of its rotation. In the integrated justification of the shape of the bore-hole cross-section both from the view point of the blasting action efficiency and from the view point of the rational bit design, consideration should be given to the ratio of the number of cutters to the number of the bore-hole corners. In this case, the fractional ratio of the number of cutters (more than one) to the number of corners of the bore-hole leads to a decrease in the range of the axial force, and their whole ratio leads to exclusion of torque deflecting the bit from the axis of its rotation.

The proposed criteria for the shape of the cross-section can be used for a multifactor analysis of the design of a drilling tool, taking into account the shape of the cross-section of an explosive cavity created by it for fracturing of rock, and also for consideration of the influence on the duty and power parameters of the drilling process, which are interrelated with the oscillatory processes occurring both in the rock [13] and in the structure of the drilling tool and machine tool [14], which in turn affects the strength of the elements of their design [15].

\section{References}

1. S. Bhandari, Proceedings of Rock Fragmentation by Blasting, 511 (2013)

2. A. José, P. Sanchidrián, International Journal for Blasting and Fragmentation, 4, 1 (2000)

3. S.H. Cho, Y. Nakamura, B. Mohanty, H.S Yang, K. Kaneko, Engineering Fracture Mechanics, 75, 3966 (2008)

4. Finn Ouchterlony, Mats Olsson, S. O. Båvik, International Journal for Blasting and Fragmentation, 4, 55 (2000)

5. Zong-Xian Zhang, Rock Fracture and Blasting, 6, 217 (2016)

6. Wang Yan-mei, Li Cheng-fang, Chongqing architecture, 11, 51 (2005)

7. Ding Dexing, Zhv. Chenghang, Trans Nonferrous Metals Soc China, 1, 188 (1999)

8. C. E. Tsoutrelis, N. Gikas, P. Nomikos, G. Exadaktylos, International Journal for Blasting and Fragmentation, 1, 445 (1997)

9. Yue Zhongwen, Guo Yang, WangXu, Chinese Journal of Rock Mechanics and Engineering, 10 (2015)

10. Yu Musong, Yang Yongqi, Yang Renshu, Zhang Qi, Explosion and shock waves, 2 (1997)

11. A. Zhukov, L. T. Dvornikov and S. M. Nikitenko, IOP Conference Series: Materials Science and Engineering, 124, 012171 (2016)

12. G. D. Buyalich, K. G. Buyalich, V. Yu. Umrikhina, IOP Conf. Series: Materials Science and Engineering, 142, 012120 (2016)

13. E.N. Pashkov, G.R. Zijakaev, M.V. Tsigankova, Applied Mechanics and Materials, 379, 91 (2013)

14. G. D. Buyalich, A. V. Anuchin, K. P. Serikov, IOP Conf. Series: Materials Science and Engineering, 127, 012012 (2016) 